\title{
Gerenciamento de resíduos sólidos orgânicos do Consórcio do Maciço de Baturité: Análise técnica e econômica da geração de biogás por aterro sanitário e usina de digestão anaeróbia
}

Management of solid organic waste of the Baturité Massif Consortium: a technical and economic analysis of biogas generation from a landfill and an anaerobic digestion plant

Hellen Luisa de Castro e Silva** ${ }^{\circ}$, Hiliene da Costa de Carvalho ${ }^{2} \odot$, Jucimar Pinto ${ }^{2} \odot$, Livya Wana Nascimento² ${ }^{2}$, Renato Mello Silva² ${ }^{(1)}$

\begin{abstract}
RESUMO
Os sistemas de recuperação de biogás contribuem para a mitigação de gases do efeito estufa, além de agregarem valor econômico ao processo de decomposiçãoanaeróbia da matéria orgânica. O presentetrabalho buscaanalisar os potenciais energéticos e a viabilidade econômica de implementação de um sistema de recuperação de biogás de aterro sanitário (cenário 1) ou de uma usina de digestão anaeróbia (cenário 2) para o Consórcio do Maciço de Baturité no estado do Ceará, Brasil. Para isso, foi estimada a projeção populacional, seguida da estimativa da produção de biogás pelo software LandGEM. Para o sistema de recuperação de biogás do aterro sanitário, foi obtida a potência ótima de 460 kW. com geração de energia máxima de 3.223 .680 kWh/ano, enquanto, para a usina de digestão anaeróbia, foram obtidos valores de potência e energia de $252 \mathrm{~kW}$ e 1.986.754,86 kWh, respectivamente. De acordo com a análise econômica, ambos os projetos possuem inviabilidade de implementação, todavia o preço da unidade de energia gerada ( $k W h$ ) é 42,42\% maior para a usina de digestão anaeróbia em relação ao do sistema de recuperação de biogás do aterro sanitário. A metodologia aplicada pode ser utilizada para estudos preliminares em outros tipos de consórcio, contribuindo para melhores gerenciamento de resíduos e aproveitamento energético de sistemas de biogás no país.
\end{abstract}

Palavras-chave: valoração energética. bioenergia; digestão anaeróbia; maciço do Baturité.

\begin{abstract}
Biogas recovery systems contribute to the mitigation of greenhouse gases, besides adding economic value to the anaerobic decomposition of organic matter. This research aims to analyze the energy potentials and economic feasibility of implementing a landfill biogas recovery system (scenario 1) and an anaerobic digestion plant (scenario 2) for the Baturité Massif Consortium in the state of Ceará, Brazil. To that end, a population projection followed by an estimate of biogas production were made using the LandGEM software. For the biogas recovery system of the landfill, an optimum power of $460 \mathrm{~kW}$ and a maximum energy generation of 3,223,680 kWh/yr were obtained, whereas the anaerobic digestion plant resulted a power and energy of $252 \mathrm{~kW}$ and 1,986,754.86 kWh respectively. According to the economic analysis, both projects are unfeasible, but the price of the energy unit generated ( $\mathrm{kWh}$ ) is $42.42 \%$ higher for the anaerobic digestion plant than for the biogas recovery system of the landfill. The methodology applied can be used for preliminary studies in other consortiums, contributing to a better waste management and energy use of biogas systems in the country.
\end{abstract}

Keywords: energy valuation; bioenergy; anaerobic digestion; Baturité Massif.

\section{INTRODUÇÃO}

De acordo com dados do Panorama dos Resíduos Sólidos no Brasil, a geração de resíduos sólidos urbanos (RSU) no Brasil é de 216.629 toneladas por dia, estimando-se uma coleta diária de 199.311 toneladas (ABRELPE,
2017). Nessa linha, abre-se espaço para a criação de aterros sanitários, os quais apresentam uma opção menos agressiva ao meio ambiente em relação à implementação de lixões e aterros controlados e são eficientes e adaptáveis de acordo com o tamanho a ser atendido (ELK, 2007). O armazenamento

'Universidade de Gante - Gante, Bélgica.

¿Universidade Federal de Itajubá - Itajubá (MG), Brasil

*Autora correspondente: hellen-luisa@hotmail.com

Conflitos de interesse: Os autores não declaram nenhum conflito de interesse

Financiamento: Coordenação de Aperfeiçoamento de Pessoal de Nível Superior (CAPES).

Recebido: 04/12/2019 - Aceito: 16/09/2O2O - Reg. ABES: 20200155 
desses resíduos em aterros possibilita a degradação da matéria orgânica em meio à ausência de oxigênio, caracterizando o processo de digestão anaeróbia. Como resultado, há a formação de gases, como o metano $\left(\mathrm{CH}_{4}\right)$, com 60-70\% em sua composição, dióxido de carbono $\left(\mathrm{CO}_{2}\right)$, com 30-40\%, seguidos de gases traço como nitrogênio $\left(\mathrm{N}_{2}\right)$, hidrogênio $\left(\mathrm{H}_{2}\right)$, sulfeto de hidrogênio $\left(\mathrm{H}_{2} \mathrm{~S}\right)$, amônia $\left(\mathrm{NH}_{3}\right)$ e vapor d'água $\left(\mathrm{H}_{2} \mathrm{O}\right)$, presentes em menores rendimentos (COSTA GOMEZ, 2013).

É necessário realçar que a emissão direta de $\mathrm{CH}_{4}$ no meio ambiente é preocupante uma vez que sua molécula possui efeito estufa 28 vezes maior que o $\mathrm{CO}_{2}$ (GREENHOUSE GAS PROTOCOL, 2016). Nessa esfera, a implementação desses sistemas possibilita a valoração energética do produto gerado (PAGLIUSO; REGATTIERI, 2008). Atualmente, existem vários estudos envolvendo estimativas de geração desses gases por meio de projeções populacionais e residuais para alguns municípios brasileiros, todavia é necessário realçar que grande parte dessas cidades não possui condições financeiras de gerir um aterro sanitário. Nesse sentido, as prefeituras municipais têm procurado a possibilidade da criação de consórcios entre municípios. Como vantagens, há a diminuição de custos operacionais, a otimização do uso de equipamentos e mão de obra, a redução de área utilizada e a diminuição de possíveis contaminações ambientais, além da maior facilidade de fiscalização (SUZUKI; GOMES, 2009).

Diante disso, o presente trabalho possui como objetivo realizar uma análise técnica e econômica da estimativa teórica da produção energética por meio de dois cenários para o Consórcio do Maciço de Baturité, formado pelas cidades de Acarape, Aracoiaba, Aratuba, Guaramiranga, Capistrano, Barreira, Baturité, Redenção, Pacoti, Palmácia, Ocara, Mulungu e Itapiúna, presentes no estado do Ceará. O governo do estado do Ceará, por meio da Secretaria do Meio Ambiente, formalizou o consórcio para a gestão integrada de resíduos sólidos com os municípios do Maciço de Baturité (DIÁRIO DO NORDESTE, 2018). Com a presença e a contribuição dos prefeitos da região, o consórcio prevê uma série de ações para viabilizar a gestão integrada desses municípios (DIÁRIO DO NORDESTE, 2018). Para a presente pesquisa, o primeiro cenário consiste na implementação de um sistema de recuperação de biogás para um aterro sanitário, enquanto o segundo abrange a implementação de uma usina de digestão anaeróbia.

Com base nisso, foram realizadas projeções populacionais e de geração de resíduos, seguidas de estimas da produção de biogás pelo software LandGEM para o aterro sanitário, aliadas a um projeto de dimensionamento de um sistema de digestão anaeróbia para o consórcio. A aplicação desse software é reportada também nos estudos de Barros, Tiago Filho e Silva (2014), Fernandes e Santos (2018) e Castro e Silva et al. (2020) para a estimativa da produção de gases em projetos de aterros sanitários. Para a implementação dos sistemas, foram efetuadas análises de viabilidade econômica, utilizando-se as metodologias de valor presente líquido (VPL) e custo nivelado de energia (LCOE) de maneira a apresentar uma solução integrada de gerenciamento dos resíduos gerados para o presente consórcio. É importante ressaltar que o método utilizado pode ser reproduzido para outros consórcios ou grupo de municípios, resultando em mais estudos energéticos e econômicos para a tecnologia de biogás, além do fornecimento de dados para a literatura, e contribuindo para a disseminação dessa tecnologia, a qual ainda é escassa no país.

\section{METODOLOGIA}

\section{Caracterização da área de estudo}

Para o presente estudo, foi escolhida a Região do Maciço de Baturité (Figura 1), localizada no estado do Ceará (CE) e composta dos municípios: Acarape, Aracoiaba, Aratuba, Guaramiranga, Capistrano, Barreira, Baturité, Redenção, Pacoti, Palmácia, Ocara, Mulungu e Itapiúna. A região ocupa área de área de $3.707,26 \mathrm{~km}^{2}$ e, por meio do consórcio formalizado, será construída uma central receptora de resíduos, composta de galpão, setores de recepção de triagem, apoio e controle, áreas verdes, compostagem e resíduos de construção civil (DIÁRIO DO NORDESTE, 2018).

\section{Projeção populacional}

Para a determinação da população total do consórcio, foram coletados dados de população dos últimos censos (1990, 2000 e 2010) (IBGE, 2019) e realizou-se a estimativa da população, de acordo com a função logística, para os municípios de Palmácia, Acarape, Aracoiaba, Ocara, Mulungu e Itapiúna, em razão de essa função apresentar crescimento limitado, ou seja, não crescer indefinidamente (CASTRO E SILVA et al., 2020). A taxa decrescente de crescimento foi aplicada para as cidades de Aratuba, Pacoti, Redenção, Baturité e Barreira uma vez que se observou que, à medida que as cidades cresciam, suas taxas de crescimento tornavam-se menores no período de 1970 a 2010 (IBGE, 2019). Nesse sentido, observa-se que as populações estão tendendo, assintoticamente, a um valor de saturação (VON SPERLING, 2014).

Para as cidades de Capistrano e Guaramiranga, foi observada uma taxa de crescimento negativa da quantidade de habitantes no período de 1970 a 2010 (IBGE, 2019). Com base nisso, as projeções de suas populações foram obtidas por meio de regressão linear simples com o auxílio da ferramenta Solver ${ }^{\circledR}$ do Microsoft Excel ${ }^{\boxplus}$. De acordo com Ragsdale (2009), em situações em que se faz necessário identificar e analisar uma ou mais variáveis independentes a uma variável dependente, pode-se empregar o modelo de análise de regressão.

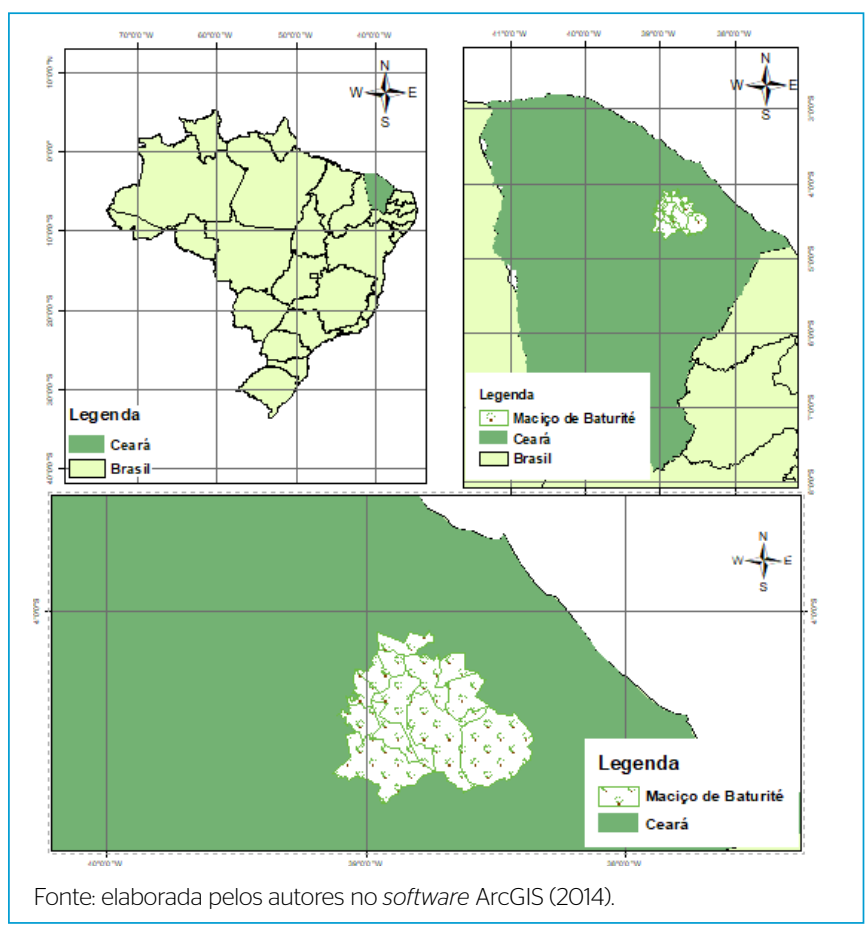

Figura 1 - Mapa de Localização da área de estudo. 


\section{Projeção da geração de resíduos}

Para a obtenção dos resíduos gerados ao longo do tempo de projeção de 20 anos, foram utilizados os índices de geração de resíduos, retirados do Sistema Nacional de Informações Sobre Saneamento (SNIS, 2019) e acrescidos de 1\% no valor para cada ano posterior (Equação 1) (BARROS, 2012). Os índices per capita são expostos na Tabela 1.

$R_{t}=365 \times\left(I_{\text {ger.t. }}\right) \times P_{t} / 1000$

Em que:

$R_{t}=$ é a geração de resíduos no ano $t$;

$P_{t}=$ é a população no ano $t$;

$I_{\text {gert }}=$ é o índice de geração de resíduos no ano $t$, em kg.hab ${ }^{-1} \cdot \mathrm{d}^{-1}$.

\section{Estimativa da geração de biogás para o aterro sanitário do consórcio e cálculo do potencial energético: cenário 1}

Neste cenário, os dados de geração de resíduos foram aplicados ao software LandGEM (versão 3.02), o qual estimou a produção de biogás para o aterro contabilizando os resíduos totais obtidos para o consórcio para o período de 20 anos. O modelo é utilizado com base em uma equação de primeira ordem, como mostrado na Equação 2. Dois parâmetros importantes são utilizados na modelagem, a constante da taxa de produção de metano, $\mathrm{k}\left(\right.$ ano $\left.^{-1}\right)$, e o potencial de produção de metano, $\mathrm{L}_{0}\left(\mathrm{~m}^{3} / \mathrm{mg}\right)$ (FALLAHIZADEH et al., 2019).

$Q_{C_{4}}=\sum_{i=1}^{n} \sum_{j=0,1}^{1} k L o\left(\frac{M i}{10}\right) e^{-k t_{i j}}$

Com base na geração anual de metano, foi calculada a potência passível de ser obtida durante a vida útil de implementação do projeto por meio da Equação 3, de acordo com Barros (2012).

Tabela 1 - Índice per capita dos municípios pertencentes ao Consórcio do Maciço de Baturité*.

\begin{tabular}{l|c|c} 
Município & $I_{\text {gert. }}\left(\mathrm{kg} \cdot \mathrm{hab}^{-1} \cdot \mathrm{d}^{-1}\right)$ & Ano \\
\hline Palmácia & 1,1100 & 2013 \\
\hline Acarape & 0,3436 & 2014 \\
\hline Aracoiaba & 0,6034 & 2017 \\
\hline Ocara & 0,6553 & 2017 \\
\hline Mulungu & 0,2745 & 2017 \\
\hline Itapiúna & 0,2000 & 2015 \\
\hline Aratuba & 0,6441 & 2015 \\
\hline Pacoti & 0,2300 & 2017 \\
\hline Redenção & 0,3300 & 2017 \\
\hline Baturité & 0,3890 & 2015 \\
\hline Barreira & 0,3030 & 2015 \\
\hline Capistrano & 0,5500 & 2011 \\
\hline Guaramiranga & 0,5500 & 2011 \\
\hline
\end{tabular}

*É necessário realçar que, em virtude da ausência de informações para o ano mais recente, 2017, foram utilizados os índices per capita dos anos de 2013, 2014 e 2015 para a realização dos cálculos de projeção.

Fonte: SNIS (2019).
$P=Q_{C H_{4}} \times E \times E_{C} \times P C_{C H_{4}} \times(1 / 31.536 .000) \times(1 / 1000)$

Os dados de entrada para o software LandGEM e demais equações apresentadas, além dos anos de início e fechamento do aterro, podem ser visualizados na Tabela 2.

É necessário realçar que a produção de gás em aterros sanitários atinge seu pico no ano posterior ao seu fechamento, passando a declinar após essa fase. A partir de então, a produção residual resulta em quantidades menores de produção de biogás pelos microrganismos, os quais utilizam a degradação do material orgânico advindo dos resíduos depositados nos anos anteriores, contribuindo para a variação da energia disponível pelo gás produzido de ano para ano. Com isso, o investimento deve ser realizado no primeiro ano e a planta deve ser dimensionada para uma descarga constante de biogás ou de variação conhecida. Portanto, deve ser escolhida uma potência ótima para a implementação do sistema, a qual está associada a um tempo de disponibilidade, como especificado no método apresentado por Silva et al. (2017). Este é semelhante ao usado em pequenas centrais hidrelétricas ( $\mathrm{PCH}$ ) para obter a melhor utilização da energia, que se baseia na determinação de uma curva de permanência com certa potência (descarga de biogás) em sua permanência (SILVA et al., 2017).

Nesse sentido, a porcentagem de tempo em que uma descarga é igual ou menor estará disponível nos anos seguintes após o início da operação do aterro. Com base nessa curva, são calculados a energia gerada, a receita da venda de energia e os custos de implementação, operação e manutenção, além do benefício gerado por cada potência para, posteriormente, a potência advinda do máximo benefício econômico ser escolhida (SANTOS; BARROS; TIAGO FILHO, 2018).

Para a aplicação dessa metodologia, foram estabelecidos intervalos de potências, partindo-se dos valores mínimo e máximo $\left(P_{\text {min }}\right.$ e $\left.P_{\text {máx }}\right)$ obtidos pela Equação 3. Em seguida, foram geradas duas equações de ajuste de acordo com as fases de produção do gás pelo aterro, intituladas "Potência da fase de entrada dos resíduos" e "Potência da fase de produção residual". Com elas foram obtidos os tempos inicial e final $\left(t_{i}, t_{f}\right)$ pela interseção entre cada valor de potência com a curva de potência disponível para as fases de produção. Em seguida, foi calculado o incremento de energia $(\Delta \mathrm{E})$ (Equação 4$)$ e, por meio de um varrimento da curva, foi obtida a potência ótima $\left(P_{o t m}\right)$, a qual produz a máxima energia passível de ser alcançada pelo sistema. A curva do $\Delta \mathrm{E}$, para cada valor de potência, é dada pela Equação 4, enquanto a máxima energia suscetível de geração pelo sistema é fornecida pela Equação 5.

$\Delta E=P \times\left(t_{f}-t_{i}\right) \times 8760 \times f_{c} / 10^{6}$

$E=P_{\text {otm }} \times 8760 \times f_{c}$

Em que:

$\Delta E=$ incremento de energia, em gigawatt-hora (GWh);

$P=$ potência disponível de acordo com os tempos das fases de produção, em quilowatt $(\mathrm{kW})$;

$t_{i}=$ tempo inicial em anos;

$t_{f}=$ tempo final em anos;

8760 = número de horas presentes em um ano;

$f_{c}=$ fator de capacidade de $80 \%$. 


\section{Estimativa da produção de biogás pela usina de digestão anaeróbia e cálculo do potencial energético: cenário 2}

Neste cenário, há o encaminhamento dos resíduos totais para uma usina de digestão anaeróbia por batelada para o presente consórcio. A metodologia é aplicada de acordo com o Programa de Pesquisas em Saneamento Básico (PROSAB, 2003), considerando-se a produção de resíduos sólidos orgânicos (RSO) para a população no ano de 2033 e os seguintes dados, expostos na Tabela 3.

\section{Determinação da massa de resíduos sólidos orgânicos}

Para este caso, foram considerados os RSO produzidos em áreas urbanas, como especificado na Equação 6:

$M_{R S O-b u}=P P_{R S O-b u} x P$

Em que:

$M_{R S O-b u}=$ massa diária de RSO, em base úmida $\left(\mathrm{kg} \cdot \mathrm{d}^{-1}\right)$;

$P P_{R S O-b u}=$ produção per capita de RSO, em base úmida, de $0,574 \mathrm{~kg} \cdot \mathrm{hab}^{-1} \cdot \mathrm{d}^{-1}$;

$P=$ população do consórcio projetada para o ano de 2033.

\section{Determinação da massa de demanda química de oxigênio dos resíduos sólidos orgânicos produzidos}

A massa diária de RSO em base seca e a massa diária de demanda química de oxigênio (DQO) foram determinadas pela Equação 7 e pela Equação 8.

$M_{R S O-b s}=M_{R S O-b u} \times S T$

$M_{D Q O-b s}=M_{R S O-b s} \times D Q O$

Em que:

$M_{R S O-b s}=$ massa diária de RSO, em base seca $\left(\mathrm{kg} . \mathrm{d}^{-1}\right)$;
$S T=$ concentração de sólidos totais na massa dos RSO, de $20 \%$.

$M_{D Q O-b s}=$ massa diária de DQO dos RSO, em base seca $\left(\mathrm{kg} . \mathrm{d}^{-1}\right)$;

DQO = demanda química de oxigênio dos RSO, em base seca de 30\% usualmente, conforme PROSAB (2003).

\section{Determinação do volume total necessário}

Para o tratamento de RSO com $20 \%$ de sólidos totais (ST), em média, adotou-se a carga orgânica de $70 \mathrm{~kg}$ de DQO. $\mathrm{m}^{-3}$ de reator, conforme PROSAB (2003). Nesse sentido, o volume total para o consórcio é fornecido pela Equação 9.

Tabela 3 - Parâmetros para o dimensionamento do sistema de digestão anaeróbia do Consórcio do Maciço de Baturité.

\begin{tabular}{|c|c|}
\hline Parâmetro & Valor \\
\hline Produção per capita de RSO & $0,574 \mathrm{~kg} \cdot \mathrm{hab}^{-1} \cdot \mathrm{d}^{-1}$ \\
\hline Teor de umidade dos RSO & $80 \%$ \\
\hline Porcentagem de sólidos totais & $20 \%$ \\
\hline Peso específico do resíduo & $950 \mathrm{kgfm}^{-3}$ \\
\hline Tempo de retenção de sólidos & 200 dias \\
\hline Tipo de reator & Batelada \\
\hline Parâmetro de dimensionamento & DQO \\
\hline Vida útil do sistema & 15 anos* \\
\hline
\end{tabular}

RSO: resíduos sólidos orgânicos; DQO: demanda química de oxigênio; *de acordo com a US Environmental Protection Agency - EPA (2005), os padrões da Lei do Ar Limpo (CAA) são baseados nos requisitos para aterros sanitários de RSU estabelecidos pela CAA, incluindo o New Source Performance Standards (NSPS, ou Novos Padrões de Desempenho da Fonte, em português)/ Emission Guidelines (EG, ou Diretrizes de Emissão, em português) e o National Emission Standards for Hazardous Air Pollutants (NESHAP, ou Padrões Nacionais de Emissão para Poluentes Perigosos do Ar, em português). Esse conjunto de parâmetros padrão produz estimativas conservadoras de emissões e é utilizado para determinar se determinado tipo de aterro está sujeito aos requisitos de controle Fonte: PROSAB (2003) e Brasil (2015).

Tabela 2 - Dados de entrada para estimativa da produção de biogás e cálculo do potencial energético do aterro sanitário.

\begin{tabular}{|c|c|c|}
\hline Parâmetros & Dados de entrada & Referências \\
\hline Ano de abertura aterro - Ano de fechamento do aterro, em anos & 2019- 2039 & - \\
\hline Ano do cálculo - ano inicial de aceitação de resíduos ( $n$ ) & - & - \\
\hline Eficiência de conversão do biogás - $E_{C}$, em \% & $55,50 \%$ & $\begin{array}{c}\text { Santos, Barros e Tiago Filho (2018), calculado } \\
\text { por meio da média aritmética dos estudos de } \\
\text { Silva, Freitas e Candiani (2013) }\end{array}$ \\
\hline Eficiência da turbina/motor (E) considerando-se a microturbina a gás (MTG), em \% & $25 \%$ & Santos et al. (2015) \\
\hline Idade da j-ésima seção de massa residual $M_{i}$ aceita no i-ésimo ano $\left(t_{i}\right)$, em anos decimais & - & - \\
\hline Incremento de tempo de 1 ano $(i)$ & 1 ano & Barros (2012) \\
\hline Incremento de tempo de 0,1 ano $(j)$ & 0,1 ano & EPA (2005) \\
\hline Massa de resíduos aceita no i-ésimo ano $(M)$, em mg & - & - \\
\hline Número de segundos em 1 ano, em segundos/ano & 31.536 .000 & Barros (2012) \\
\hline Poder calorífico do metano $\left(P_{\mathrm{CCH} 4}\right)$, em J/m³ $\mathrm{CH}_{4}$ & $35,53.10^{6} \mathrm{~J} / \mathrm{m}^{3} \mathrm{CH}_{4}$ & Barros (2012) \\
\hline Potência disponível a cada ano $(P)$, em kW & - & - \\
\hline Potencial de geração de $\mathrm{CH}_{4}$ (padrão CAA convencional') em m³. $\mathrm{mg}^{-1}$ & $170 \mathrm{~m}^{3} \cdot \mathrm{mg}^{-1}$ & EPA (2005) \\
\hline Taxa constante de geração de $\mathrm{CH}_{4}(k)$, em ano-1 & $0,05 \mathrm{ano}^{-1}$ & EPA (2005) \\
\hline Transformação da unidade, de J/s para kW & $1 / 1.000$ & Barros (2012) \\
\hline Vazão de metano a cada ano $\left(Q_{C H 4}\right)$, em $\mathrm{m}^{3} \mathrm{CH}_{4} /$ ano & - & - \\
\hline
\end{tabular}

Fonte: elaborada pelos autores. 
$V_{T}=\left(M_{D Q O-b s} x T R S\right) \times C_{V^{-1}}$

Em que:

$V_{T}=$ volume do reator, $\mathrm{em} \mathrm{m}^{3}$;

TRS = tempo de retenção dos sólidos, de 200 dias.

$C_{V-1}=$ carga orgânica volumétrica, de $70 \mathrm{~kg}$ de DQO. $\mathrm{m}^{-3}$

\section{Determinação do tempo de carregamento}

O tempo de carregamento de cada reator é dado pela Equação 10.

$T_{C R}=V_{R} / V_{R D}$

Em que:

$T_{C R}=$ tempo de carregamento de cada reator, por dia;

$V_{R}=$ volume de cada reator $\left(\mathrm{m}^{3}\right)$;

$V_{R D}=$ volume requerido diariamente $\left(\mathrm{m}^{3}\right)$.

\section{Dimensionamento do leito filtrante e do gasômetro do reator}

Para o presente caso, foram considerados os volumes do leito filtrante e do gasômetro de 4,5\% e 5,5\% do volume total do reator. Esses volumes foram calculados de acordo com a Equação 11 e a Equação 12.

$V_{L F}=V_{T} \times 4,5 \%$

$V_{G}=V_{T} \times 5,5 \%$

Em que:

$V_{L F}=$ volume do leito filtrante $\left(\mathrm{m}^{3}\right)$;

$V_{G}=$ volume do gasômetro $\left(\mathrm{m}^{3}\right)$.

Após o dimensionamento do sistema, calculou-se o potencial energético do sistema, de maneira a analisar a viabilidade econômica da implementação do projeto.

\section{Determinação do potencial energético}

Para o caso em estudo, o potencial energético é expresso em termos de volume de gás metano $\left(\mathrm{CH}_{4}\right)$ em função da massa de DQO produzida, levando-se em consideração a equação estequiométrica (Equação 13).

$\mathrm{CH}_{4}+2 \mathrm{O}_{2} \rightarrow \mathrm{CO}_{2}+2 \mathrm{H}_{2} \mathrm{O}$

A quantificação de massa e vazão de $\mathrm{CH}_{4}$ produzidos é dada pelas Equações 14 e 15:

$M_{C H_{4}}=M_{D Q O} \times E_{T} \times F_{C}$

$V_{\mathrm{CH}_{4}}=\mathrm{M}_{\mathrm{CH}_{4}} /{\mathrm{M} \cdot \mathrm{E}_{\mathrm{CH}_{4}}}_{\mathrm{H}}$

Em que:

$M_{C H 4}=$ massa de gás metano produzida por dia, em kg.d $\mathrm{d}^{-1}$;

$M \cdot E_{C H 4}=$ massa específica do gás metano, de $0,66 \mathrm{~kg} \cdot \mathrm{m}^{-3}$;

$M_{D Q O}=$ massa de DQO, em kg.d $\mathrm{d}^{-1}$;

$V_{C H 4}=$ vazão de metano produzida por dia, $\mathrm{em} \mathrm{m}^{3} \cdot \mathrm{d}^{-1}$
$E_{T}=$ eficiência de transformação da massa de DQO, de 0,8 \%;

$F_{C}=$ fator de conversão de massa de DQO em massa de gás metano, de $0,25 \%$.

A potência e a energia proporcionadas pelo sistema são obtidas pela Equação 16, de acordo com Barros (2012), e pela Equação 17, de acordo com Ribeiro et al. (2016).

$P=Q_{C_{4}} \times E \times E_{C} \times P C_{C H_{4}} \times(1 / 31.536 .000) \times(1 / 1000)$

$E=P \times 7884$

Em que:

$Q_{C H 4}=$ vazão de metano produzida por ano $\left(\mathrm{m}^{3} \mathrm{CH}_{4} \cdot\right.$ ano $\left.^{-1}\right)$;

$E_{C}=$ eficiência de coleta do biogás pelo biodigestor, de $86 \%$, conforme os valores de perda de $14 \%$ de $\mathrm{CH}_{4}$ observados na prática de acordo com Keller e Hartley (2003), citado por Brasil (2015);

$E=$ eficiência da turbina/motor, de 33\% (CETESB, 2006);

$31.536 .00=$ número de segundos em 1 ano (s/ano);

$1 / 1.000=$ para a transformação da unidade de $\mathrm{J} / \mathrm{s}$ para $\mathrm{kW}$;

7.884 = número total de horas de funcionamento no ano, descontando-se $10 \%$ para a parada do sistema.

\section{Determinação de viabilidade econômica para a implementação alternativa dos cenários}

No intuito de realizar uma análise completa dos aproveitamentos energéticos passíveis de serem obtidos pela recuperação do biogás advinda do aterro sanitário, bem como da usina de digestão anaeróbia, foi realizado um estudo de viabilidade econômica da implementação dos projetos considerados. Para essa análise, foram considerados o VPL e o LCOE passíveis de serem obtidos durante os 20 anos e 15 anos de funcionamento do sistema de recuperação de biogás do aterro sanitário e da usina de digestão anaeróbia, respectivamente, conforme a Equação 18 e a Equação 19 (SAYIGH, 2012; BRASIL, 2014; VÖGELI, 2014).

O VPL baseia-se na rede de fluxos de caixa futuros resultantes do projeto considerando-se um desconto desde o início do investimento. Se o VPL for maior que zero, o projeto será aceito; caso contrário, deverá ser rejeitado (YANG et al., 2012; ŽIŽLAVSKÝ, 2014). O LCOE determina qual deve ser o preço estabelecido, por unidade de eletricidade gerada, para recuperar os custos da vida útil do sistema. Com base nele, são levados em consideração o investimento, os custos de manutenção e o custo do combustível para o sistema (se houver), além da taxa do mercado estabelecida, como exposto na Equação 20 (SAYIGH, 2012).

$V P L=\sum_{t=0}^{n} C F_{t} /(1+r)^{t}$

Em que:

$V P L=$ valor presente líquido, em reais $(\mathrm{R} \$)$;

$C F_{t}=$ fluxo de caixa líquido gerado pelo projeto no ano $t$, em reais (R\$);

$G_{0}=$ investimento inicial, em reais (R\$);

$T I R=$ taxa interna de retorno, em \%;

$r=$ taxa de juros, em \%. 
$L C O E=\frac{\sum_{t=0}^{n} \frac{I_{t}+M_{t}+F_{t}}{(1+i)^{t}}}{\sum_{t=0}^{n} \frac{E(t)}{(1+i)^{t}}}$

Em que:

$I_{t}=$ investimento total no ano $\mathrm{t}$, em reais $(\mathrm{R} \$)$;

$M_{t}=$ despesas de operação e manutenção no ano t, em reais (R\$);

$F_{t}=$ despesas com combustíveis para o ano $t$, em reais (R\$);

$E(t)=$ energia gerada no ano $t$, em quilowatt-hora $(\mathrm{kWh}) ;$

$i=$ taxa de juros do mercado, em \%;

$t=$ ano de operação, em ano;

$n=$ vida útil esperada para o sistema de energia, em anos.

Para os custos de operação e manutenção (O\&M) e venda de energia, foram considerados, respectivamente, $5 \%$ do investimento inicial (BRASIL, 2015) e $\mathrm{R} \$ 0,1879$ para o preço de venda do $\mathrm{kWh}$, de acordo com o preço do leilão de energia para as fontes térmicas a biomassa (ANEEL, 2019). O valor do investimento para a instalação do sistema de recuperação do biogás no aterro sanitário foi estabelecido de acordo com o trabalho de Santos et al. (2015), ao utilizarem microturbinas a gás (MTG) com eficiência de $25 \%$ e emissões de $\mathrm{NO}_{x}<9 \mathrm{ppm}$, por meio da Equação $20\left(\mathrm{R}^{2}=1\right)$, valor atualizado pela calculadora do cidadão do Banco Central do Brasil, índice (IGP-M) (BCB, 2019).

$I=0,012 P+0,520$

Em que:

$I$ = investimento total, em milhões de reais (R\$), considerando-se o comprimento do gasoduto igual a $500 \mathrm{~m}$;

$P=$ potência, em quilowatts $(\mathrm{kW})$.

Os custos de investimento e operação para uma usina de digestão anaeróbia variam de acordo com o tamanho do digestor, do substrato utilizado e da sua preparação, além da técnica de implementação (custos de operação). Baseado em estudos anteriores, utilizou-se o valor de investimento máximo advindo de IRENA (2012), cujo valor, de USD 6.500/kW, foi reajustado para o ano de 2019 com base no cálculo da inflação do dólar. Em seguida, o valor foi transformado em reais (R\$) com base no valor médio de conversão do dólar para o real no ano de 2019 (CLUBE DOS POUPADORES, 2019; INVESTING, 2019).

A Tabela 4 e a Tabela 5 expõem os parâmetros de entrada para a análise de viabilidade econômica da implementação do sistema de recuperação de biogás no aterro sanitário e da usina de digestão anaeróbia dos RSO, analisados a uma

Tabela 4 - Parâmetros de entrada para a análise de viabilidade econômica da implementação do sistema de recuperação do biogás no aterro sanitário.

\begin{tabular}{c|c} 
Parâmetro & Valor em reais (R\$) \\
Investimento/kW & $723.730,99$ \\
\hline Investimento total & $7.563 .805,10$ \\
\hline Custo de manutenção e operação (M\&O) & $378.190,26$ \\
\hline Preço do kWh & 0,1879 \\
\hline Receita anual & $605.729,47$ \\
\hline
\end{tabular}

Fonte: elaborada pelos autores. taxa de juros de 8,24\% ao ano (CASTRO E SILVA et al., 2021). De acordo com esses autores, essa taxa é proveniente da junção da taxa básica de juros brasileira (taxa anual de 6,4\% - BCB, 2019) a um fator de risco de investimento $(1,84 \%$ adotado), totalizando $8,24 \%$ por ano.

\section{RESULTADOS E DISCUSSÃO}

\section{Análise da projeção populacional e de geração de resíduos}

Aplicando-se as metodologias de crescimento populacional e de geração de resíduos, têm-se as projeções de população e de resíduos para o consórcio expostas na Figura 2 e na Figura 3, respectivamente.

O comportamento quantitativo da população não segue o mesmo padrão, uma vez que cada município possui características distintas resultantes do

Tabela 5 - Parâmetros de entrada para a análise de viabilidade econômica da implementação da usina de digestão anaeróbia no consórcio.

\begin{tabular}{c|c} 
Parâmetro & Valor em reais (R\$) \\
Investimento/kW & $32.128,614 / \mathrm{kW}$ \\
\hline Investimento total & $8.096 .357,18$ \\
\hline Custo de manutenção e operação (M\&O) & $404.817,86$ \\
\hline Preço do kWh & 0,1879 \\
\hline Receita anual & $373.311,24$ \\
\hline
\end{tabular}

Fonte: elaborada pelos autores.

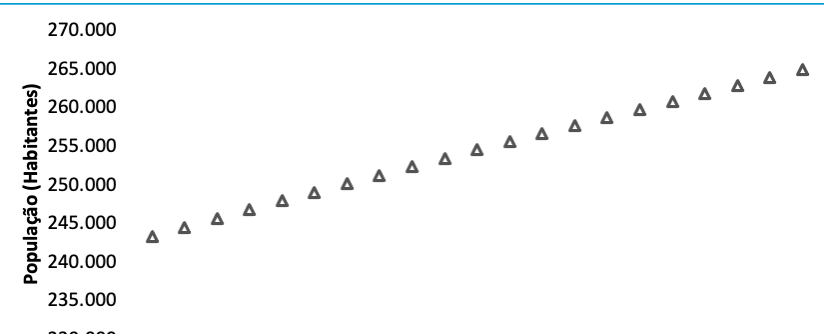

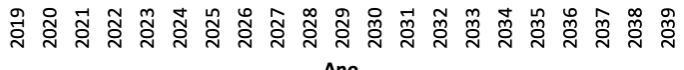 \\ Ano}

Fonte: elaborada pelos autores

Figura 2 - Projeção populacional para o período de 2019-2039 para o Consórcio do Maciço de Baturité.

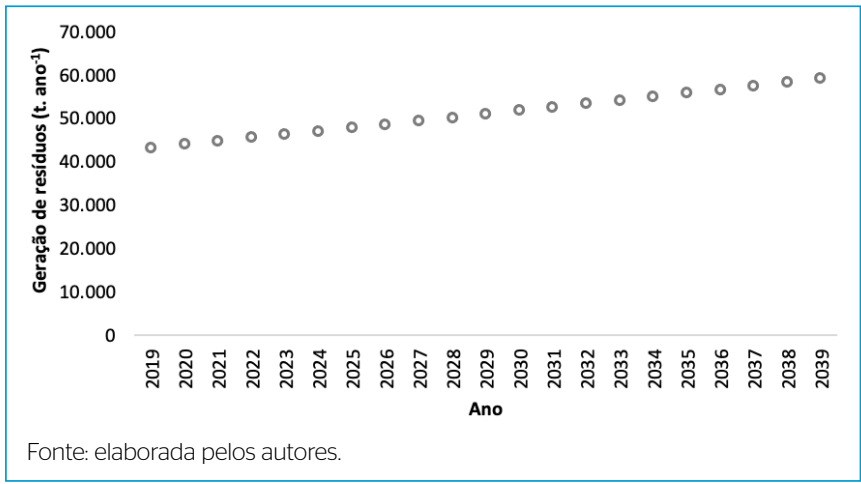

Figura 3 - Projeção da geração de resíduos sólidos orgânicos para o período de 2019-2039 para o Consórcio do Maciço de Baturité. 
crescimento ou decrescimento de sua população. O município de Guaramiranga (CE) possui decréscimo de cerca de 702 habitantes para o intervalo de tempo analisado. Diferentemente, na cidade de Baturité (CE), ocorre aumento populacional de pouco mais de 9.909 habitantes. O contraste de crescimento populacional entre essas duas últimas cidades, bem como as menores taxas de crescimento dos municípios de Mulungu, Pacoti, Aratuba, Palmácia, Ocara, Capistrano, Itapiúna, Barreira, Baturité, Aracoiaba e Redenção, resultam em aumento da população total do consórcio de somente $9 \%$ durante os 20 anos de projeção, tal como exposto na Figura 2, a qual representa a soma dos valores das populações para cada município.

A taxa de geração de resíduos de todo o consórcio oscila entre 7,47 e 8,49\% para todo o período analisado, representando a soma dos valores dos resíduos gerados para cada município (Figura 3). Os municípios de Baturité, Aracoiaba e Ocara possuem alto crescimento da geração de resíduos para o período analisado em razão do maior crescimento populacional, bem como por apresentarem maior concentração de população na área urbana (IBGE, 2019). Em contraste a esses dados, as cidades de Pacoti e Guaramiranga apresentam geração inferior a 1.400 t/ano. Apesar de esses últimos municípios, além das cidades de Capistrano, Redenção, Aratuba, Barreira, Acarapé, Itapiúna, Mulungu, Pacoti e Guaramiranga, apresentarem baixos índices populacionais, a baixa geração e a existência de áreas distantes e de difícil acesso podem resultar em menor coleta de resíduos gerados (LOPES E FIGUEIREDO et al., 2019).

\section{Análise da geração de biogás produzido pelo aterro no Modelo LandGEM (US Environmental Protection Agency): cenário 1}

O gráfico da Figura 4, obtido com o software LandGEM, mostra as emissões totais de gases $\mathrm{CH}_{4}, \mathrm{CO}_{2}$, compostos orgânicos não metânicos (non-methane organic compounds - NMOC) e o total de gás, em t.ano ${ }^{-1}$, produzidos no aterro sanitário para os anos de 2019 a 2159.

Na Figura 4, é possível observar o aumento das emissões no aterro sanitário, com os valores máximos dos gases registrados no ano de 2040, com 228 t/NMOC, 5.298 t/ $\mathrm{CH}_{4}$ e 14.536 t/ $\mathrm{CO}_{2}$, aproximadamente. Posteriormente, há o decaimento da emissão de gases até atingir-se o valor nulo no ano de 2160 . Esse cenário possui comportamento similar ao retratado por Piñas et al. (2016) para um aterro sanitário da cidade de Três Corações, Minas Gerais. A curva apresentada na Figura 5 representa a potência disponível a cada ano em kW

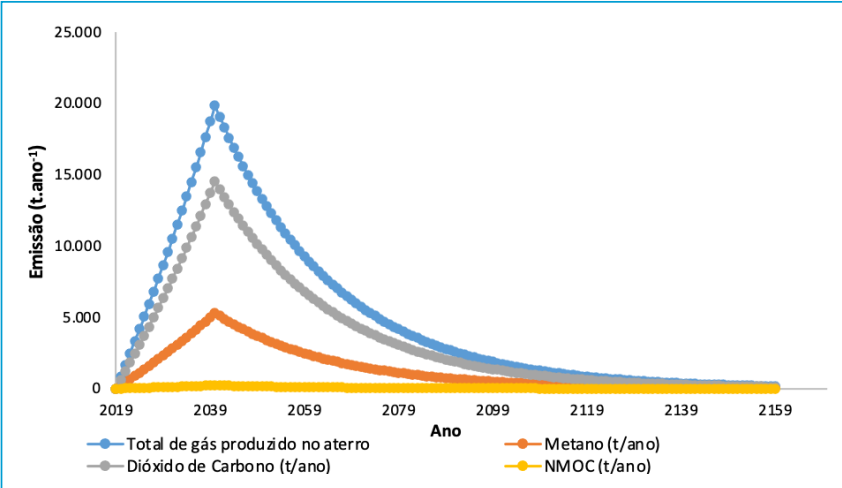

NMOC: compostos orgânicos não metânicos Fonte: elaborada pelos autores.

Figura 4 - Emissão dos gases do aterro sanitário ao longo dos anos. durante o período de funcionamento e encerramento do aterro. É possível observar as equações de ajuste correspondentes às fases de entrada dos resíduos e produção residual final, geradas antes e depois do ano de 2040, respectivamente.

De acordo com a Figura 5, a potência da fase de entrada de resíduos é dada pela Equação $21\left(y=53,503 x-108049 R^{2}=0,998\right)$, enquanto a potência da fase de produção residual é fornecida pela Equação 22 $\left(y=-25 x+2215,7 R^{2}=1\right)$. É possível constatar a produção máxima de energia no ano posterior ao fechamento do aterro. Após 2040, a degradação do material orgânico advém de resíduos depositados nos anos anteriores, resultando em menor geração de energia. Por meio de uma varredura da curva, identificou-se que a maior geração energética foi de 110,66 GWh, correspondente à potência ótima do sistema $\left(P_{o t m}\right)$ de $460 \mathrm{~kW}$, como exposto na Figura 6 .

Com base na Equação 5, foi possível obter a energia máxima de 3.223.680 $\mathrm{kWh} / \mathrm{ano}$, considerando-se $80 \%$ do fator de capacidade do sistema. Para o cálculo do investimento e da receitas passíveis de serem obtidos para esse cenário, considerou-se a energia máxima gerada pela implementação do sistema de aproveitamento energético do biogás no aterro sanitário.

\section{Análise da geração de biogás pela usina de digestão anaeróbia: cenário 2}

Na Tabela 6, são apresentados os parâmetros de dimensionamento do sistema de digestão anaeróbia para o Consórcio, de acordo com PROSAB (2003), bem como o potencial energético do sistema, considerando-se a produção residual de 258.640 habitantes obtida para o ano de 2033.

De acordo com o Ministério do Meio Ambiente - MMA (BRASIL, 2018), os resíduos orgânicos representam cerca de 50\% dos RSU gerados no País.

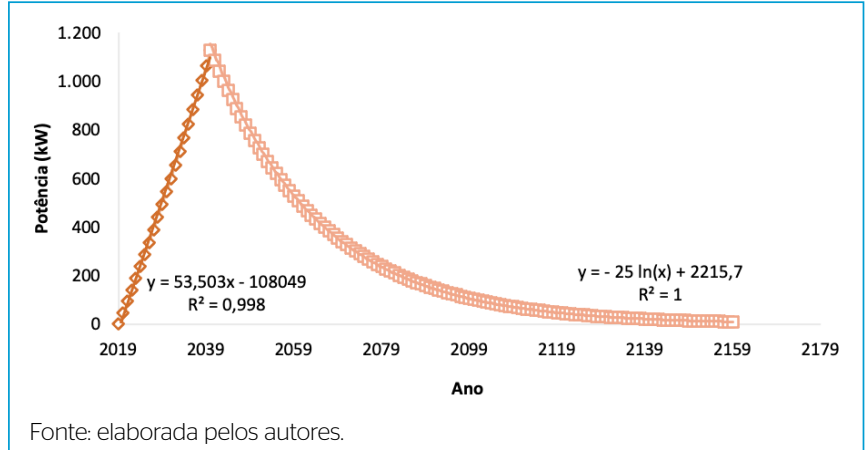

Figura 5 - Equações de ajuste dos valores de potência obtidas durante o período de geração de gases do aterro sanitário.

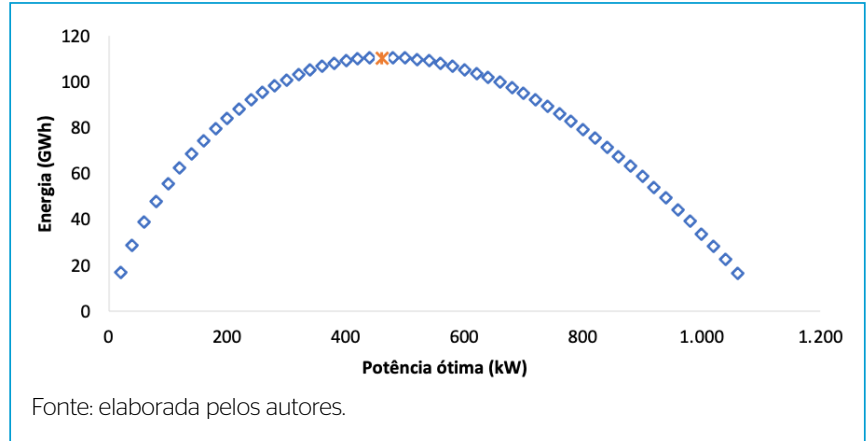

Figura 6 - Potência ótima obtida durante o período de funcionamento do aterro sanitário. 
Em 2018, foram coletadas 528 toneladas de material orgânico no Brasil pelas cooperativas e associações de catadores acompanhadas pela Associação Nacional dos Catadores e Catadoras de Materiais Recicláveis (ANCAT). Esse valor representa $0,8 \%$ de todo o material recolhido, percentual orgânico muito aquém do esperado para o volume total de 72,7 milhões de RSU gerados no Brasil. Uma alternativa para o aumento da faixa de coleta e separação desses resíduos é a implementação de consórcios de aterros sanitários, os quais acabam possuindo maiores eficiências no processo de manejo e separação dos resíduos aliadas à melhoria do processo de fiscalização (SILVA, 2015). Ainda, a implementação do processo de digestão anaeróbia torna mais atrativa o aumento da faixa de reaproveitamento dos RSO, contribuindo não somente para a diminuição da quantidade de resíduos destinados ao aterro sanitário, mas também para um tipo de tratamento da matéria orgânica.

De acordo com os resultados expostos na Tabela 6, o consórcio em estudo contabilizará a produção de, aproximadamente, 119 t/d de RSO. Nesse sentido, seriam necessários 42 reatores de $500 \mathrm{~m}^{3}$, com volumes unitários de $105 \mathrm{~m}^{3}$, tempo de carregamento de cinco dias e tempo de retenção de sólidos de 200 dias. Para essa massa de resíduos, a vazão de $\mathrm{CH}_{4} /$ ano contabilizará cerca de $800 \mathrm{mil} \mathrm{m}^{3}$, contribuindo para a potência de $252 \mathrm{~kW}$ e energia de, aproximadamente, $2 \mathrm{GWh}$ /ano. Esse resultado possibilitaria a produção de $7,68 \mathrm{kWh} /$ habitante no ano final do empreendimento, cerca de três vezes o valor do consumo de eletricidade per capita no Brasil, o qual é de 2,44 kWh/ habitante (INDEX MUNDI, 2018).

\section{Análise de viabilidade econômica para a implementação dos cenários}

A Tabela 7 expõe os valores de fluxo de caixa, VPL e LCOE obtidos na implementação dos possíveis cenários.

Tabela 6 - Parâmetros de dimensionamento e potencial energético da usina de digestão anaeróbia.

\begin{tabular}{c|c} 
Parâmetro & Valor \\
$M_{R S O-b u}$ & $118.758,99 \mathrm{~kg} \cdot \mathrm{d}^{-1}$ \\
\hline$M_{R S O-b S}$ & $23.751,80 \mathrm{~kg} \cdot \mathrm{d}^{-1}$ \\
\hline$M_{D Q O-b S}$ & $7.125,54 \mathrm{~kg} \cdot \mathrm{d}^{-1}$ \\
\hline$V_{T}$ & $21.000 \mathrm{~m}^{3}$ \\
\hline$T_{C R}$ & $5 \mathrm{dias}$ \\
\hline$V_{L F}$ & $22,5 \mathrm{~m}^{3}$ \\
\hline$V_{G}$ & $27,5 \mathrm{~m}^{3}$ \\
\hline$M_{C H 4}$ & $1.425,11 \mathrm{~kg} \cdot \mathrm{d}^{-1}$ \\
\hline$V_{C H 4}$ & $2.159,25 \mathrm{~m}^{3} \cdot \mathrm{d}^{-1}$ \\
\hline$Q_{C H 4}$ & $788.127,85 \mathrm{~m}^{3} \mathrm{CH}_{4} \cdot \mathrm{anO}$ \\
\hline$P$ & $252 \mathrm{~kW}$ \\
\hline$E$ & $1.986 .754,86 \mathrm{kWh}$ \\
\hline
\end{tabular}

Fonte: elaborada pelos autores.

Tabela 7 - Análise de viabilidade econômica para os cenários 1 e 2.

\begin{tabular}{l|c|c|c} 
& Fluxo de caixa anual (R\$) & VPL (R\$) & LCOE (R\$) \\
\hline Cenário 1 & $227.539,22$ & $-5.369 .133,15$ & 395,47 \\
\hline Cenário 2 & $-31.506,62$ & $-8.362 .130,01$ & 686,86 \\
\hline
\end{tabular}

Fonte: elaborada pelos autores.
De acordo com a Tabela 7, não há viabilidade econômica para as possíveis implementações dos sistemas em questão para o Consórcio do Maciço do Baturité. Isso pode ser constatado pelos valores de VPL negativos durante todos os períodos de funcionamento de ambos os sistemas. Para a usina de digestão anaeróbia (Cenário 2), é possível observar que as receitas anuais não cobrem os custos anuais do sistema, gerando fluxo de caixa anual negativo. Ainda, é possível notar que o custo mínimo da energia produzida pelo sistema de recuperação de biogás (Cenário 1) é 42,42\% menor em relação ao da usina de digestão anaeróbia. Isso se dá pelo fato de a energia produzida para o Cenário 1 ser, aproximadamente, 1,6 vez maior em relação à produzida pelo Cenário 2. Todavia, apesar de os resultados obtidos serem favoráveis para o primeiro cenário, é necessário realçar que o custo mínimo de energia obtido ainda representa um alto valor no mercado de energias renováveis no país.

\section{CONCLUSÃO}

O presente trabalho analisou o aproveitamento energético advindo das possíveis implementações de um sistema de recuperação energética baseado no biogás de aterro sanitário (Cenário 1) e de uma usina de digestão anaeróbia (Cenário 2) para o Consórcio do Maciço do Baturité, no estado do Ceará. Para o sistema de recuperação energética de biogás por aterro sanitário, foi obtida a potência ótima de de $460 \mathrm{~kW}$, com geração de energia máxima de 3.223.680 kWh/ano para $80 \%$ do fator de capacidade do sistema, enquanto, para a usina de digestão anaeróbia, obtiveram-se potência de $252 \mathrm{~kW}$ e energia de $1.986 .754,86 \mathrm{kWh}$, descontando-se $10 \%$ do total de horas anuais para parada do sistema. De acordo com os resultados obtidos, não há viabilidade econômica na implementação de qualquer dos sistemas, dado que o preço da unidade de energia gerada, em $\mathrm{kWh}$, é cerca de $42,42 \%$ maior para a usina de digestão anaeróbia quando comparado ao do sistema de recuperação do biogás pelo aterro sanitário. Uma solução para esse caso seria o subsídio de investimentos por parte do poder público, bem como o pagamento de valores superiores por unidade de energia limpa em $\mathrm{kWh}$, como já é realizado em vários países europeus, de maneira a viabilizar o investimento para esses sistemas. Ainda, poderá considerar-se em trabalhos futuros um cenário em que as duas alternativas sejam contempladas simultaneamente, de modo a contribuir para que novos estudos sejam realizados no presente consórcio.

\section{CONTRIBUIÇõES DOS AUTORES}

Silva, H. L. C.: Conceituação, Curadoria de Dados, Análise formal, Investigação, Metodologia, Administração do projeto, Recursos, Software, Supervisão, Validação, Visualização, Escrita - primeira redação, Escrita - revisão e edição. Carvalho, H. C.: Conceituação, Curadoria de Dados, Análise formal, Investigação, Metodologia, Administração do projeto, Recursos, Software, Validação, Visualização, Escrita - primeira redação. Pinto, J.: Conceituação, Curadoria de Dados, Análise formal, Investigação, Metodologia, Recursos, Software, Validação, Visualização, Escrita - primeira redação. Nascimento, L. W.: Conceituação, Curadoria de Dados, Análise formal, Investigação, Metodologia, Administração do projeto, Recursos, Software, Validação, Visualização, Escrita - primeira redação. 


\section{REFERÊNCIAS}

AGÊNCIA NACIONAL DE ENERGIA ELÉTRICA (ANEEL). Leilão de energia garante investimento de $R \$ 11,2$ bilhões. ANEEL, 2019. Disponível em: https://www.aneel.gov.br/sala-de-imprensa. Acesso em: 3 dez. 2019.

ASSOCIAÇÃO BRASILEIRA DE EMPRESAS DE LIMPEZA PÚBLICA E RESÍDUOS ESPECIAIS (ABRELPE). Panorama dos resíduos sólidos no Brasil. ABRELPE, 2017. Disponível em: http://abrelpe.org.br/pdfs/panorama/ panorama_abrelpe_2017.pdf. Acesso em: 1 dez. 2019

BANCO CENTRAL DO BRASIL (BCB). Calculadora do Cidadão. BACEN, 2019. Disponível em: https://www.bcb.gov.br/acessoinformacao/ calculadoradocidadao. Acesso em: 3 dez. 2019.

BARROS, R. M. Tratado sobre resíduos sólidos: gestão, uso e sustentabilidade. Rio de Janeiro: Interciência, 2012. 374 p.

BARROS, R. M.; TIAGO FILHO, G. L.; SILVA, T. R. The electric energy potential of landfill biogas in Brazil. Energy Policy, v. 65, p. 150-164, 2014. https://doi. org/10.1016/j.enpol.2013.10.028

BRASIL. Ministério do Meio Ambiente. Compostagem doméstica, comunitária e institucional de resíduos sólidos orgânicos. Brasília: MMA, 2018. 68 p.

BRASIL. Ministério do Meio Ambiente. Resíduos sólidos. Brasília: MMA, 2014. Disponível em: https://www.mma.gov.br/mma-em-numeros/residuossolidos. Acesso em: 3 dez. 2019

BRASIL. Secretaria Nacional de Saneamento Ambiental. Probiogás. Guia técnico de aproveitamento energético de biogás em estações de tratamento de esgoto. Brasília: Ministério das Cidades, 2015. 183 p.

CASTRO E SILVA, H. L.; CÓRDOVA, M. E. H.; BARROS, R. M.; TIAGO FILHO, G. L.; LORA, E. E. S.; SANTOS, A. H. M.; SANTOS, I. F. S.; ROCHA, J. V.; BOTAN, M. C. C. O.; PEDREIRA, J. R.; FLAUZINO, B. K. An agroindustry technical and economic evaluation of biogas production from swine manure in Southern Minas Gerais, Brazil. Biomass and Bioenergy, v. 148, 106013, 2021. https://doi. org/10.1016/j.biombioe.2021.106013

CASTRO E SILVA, H. L.; TOLEDO, A. L. A.; CARVALHO, B. P. A.; VILAS BOAS, L. J.; LEITE, L. E. P. Estimativa teórica da geração anual de biogás pelo aterro sanitário do consórcio CIAS. Revista DAE, São Paulo, v. 68, n. 224, p. 23-33, 2020. https://doi.org/10.36659/dae.2020.038

CLUBE DOS POUPADORES. Simulador: cálculo inflação do dólar. Clube dos Poupadores, 2019. Disponível em: https://www.clubedospoupadores.com/ simulador-inflacao-dolar. Acesso em: 15 jun. 2020.

COMPANHIA AMBIENTAL DO ESTADO DE SÃO PAULO (CETESB). Biogas Generation and Use of Energy-Landfill, Version 1.0. São Paulo: CETESB, 2006. CD-ROM

COSTA GOMEZ, C. Biogas as an energy option: an overview. In: WELLINGER, A.; MURPHY, J.; BAXTER, D. (org.). The biogas handbook: science, production and applications. Sawston: Woodhead, 2013. p. 1-16.

DIÁRIO DO NORDESTE. Municípios do Maciço de Baturité assinam consórcio de gestão de resíduos sólidos. Diário do Nordeste, 2018. Disponivel em: https://diariodonordeste.verdesmares.com.br/editorias/ regiao/online/municipios-do-macico-de-baturite-assinam-consorcio-degestao-de-residuos-solidos-1.1919374. Acesso em: 30 dez. 2019.
ELK, A. G. H. P. Redução de emissões na disposição final. Rio de Janeiro: IBAM, 2007.

FALLAHIZADEH, S.; RAHMATINIA, M.; MOHAMMADI, Z.; VAEZZADEH, M.; TAJAMIRI, A.; SOLEIMANI, H. Estimation of methane gas by LandGEM model from Yasuj municipal solid waste landfill, Iran. Methodsx, v. 6, p. 391 398, 2019. https://doi.org/10.1016/j.mex.2019.02.013

FERNANDES, G.; SANTOS, I. Avaliação do potencial energético de aterros sanitários de seis cidades brasileiras. Revista Brasileira de Energias Renováveis, Cascavel, v. 7, n. 1, p. 51-65, 2018. http://dx.doi.org/10.5380/rber.v7i1.57967

GREENHOUSE GAS PROTOCOL. Global warming potential values. 2016 Disponivel em: https://www.ghgprotocol.org/sites/default/files/ghgp/GlobalWarming-Potential-Values\%20\%28Feb\%2016\%202016\%29_1.pdf. Acesso em: 5 abr. 2020

INDEX MUNDI. Consumo de eletricidade per capita - Mundo. Index Mundi, 2018. Disponível em: https://www.indexmundi.com/ map/?v=81000\&r=xx\&l=pt. Acesso em: 14 ago. 2020.

INSTITUTO BRASILEIRO DE GEOGRAFIA E ESTATISTICA (IBGE). Sidra Banco de Tabelas Estatísticas. IBGE, 2019. Disponível em: https://sidra.ibge. gov.br/home/pimpfbr/brasil. Acesso em: 20 ago. 2019.

INTERNATIONAL RENEWABLE ENERGY AGENCY (IRENA). Biomass for power generation. Boon: IRENA Secretariat, 2012. $60 \mathrm{p}$.

INVESTING. USD/BRL - dólar americano real brasileiro. Disponível em: https://br.investing.com/currencies/usd-brl. Acesso em: 20 nov. 2019.

KELLER, J.; HARTLEY, K. Greenhouse gas production in wastewater treatment: process selection is the major factor. Water Science and Technology, v. 47, n. 12, p. 43-48, 2003. https://doi.org/10.2166/wst.2003.0626

LOPES E FIGUEIREDO, B.; LOPES, D. M. P.; PACHECO, J. M. S.; SILVA, E V. Análise do panorama da gestão dos resíduos sólidos urbanos dos municípios do maciço de Baturité. In: FÓRUM INTERNACIONAL DE RESÍDUOS SÓLIDOS, 10., 2019, p. 1-10. Anais eletrônicos [...]. João Pessoa: Instituto Venturi, 2019. Disponivel em: http://www.institutoventuri.org.br/ojs/ index.php/firs/article/view/1605. Acesso em: 10 dez. 2019.

PAGLIUSO, J. D.; REGATTIERI, C. R. Estudo do aproveitamento da energia de biogás proveniente da incineração de chorume para a geração de eletricidade. Revista Brasileira de Ciências Ambientais, Rio de Janeiro, n. 10, p. 32-38, 2008.

PIÑAS, J. A. V.; VENTURINI, O. J.; LORA, E. E. S.; OLIVEIRA, M. A.; ROALCABA, O. D. C. Aterros sanitários para geração de energia a partir da produção de biogás no Brasil: Comparação dos modelos LandGEM (EPA) e Biogás (Cetesb). Revista Brasileira de Estudos de População, Belo Horizonte, v. 33, n. 1, p. 175-188, 2016. https://doi.org/10.20947/S01O2-309820160009

PROGRAMA DE PESQUISAS EM SANEAMENTO BÁSICO (PROSAB). Digestão anaeróbia de resíduos sólidos orgânicos e aproveitamento de biogás. PROSAB, 2003. Disponível em: https:/www.finep.gov.br/images/ apoio-e-financiamento/historico-de-programas/prosab/ProsabStulio.pdf. Acesso em: 19 nov. 2019

RAGSDALE, C. T. Modelagem e análise de decisão. São Paulo: Cengage Learning, 2009. 
RIBEIRO, E. M.; BARROS, R. M.; TIAGO FILHO, G. L.; SANTOS, I. F. S.; SAMPAIO, L. C.; SANTOS, T. V.; BRAGA DA SILVA, F. G.; SILVA, A. M.; FREITAS, J. V. R. Power generation potential in posture aviaries in Brazil in the context of a circular economy. Sustainable Energy Technologies and Assessments, v. 18, p. 153-163, 2016. https://doi.org/10.1016/j.seta.2016.10.009

SANTOS, I. F. S.; BARROS, R. M.; TIAGO FILHO, G. L. Economic study on LFG energy projects in function of the number of generators. Sustainable Cities and Society, v. 41, p. 587-600, 2018. https://doi.org/10.1016/j.scs.2018.04.029

SANTOS, I. F. S.; VIEIRA, N.; BARROS, R. M.; TIAGO FILHO, G. L. Uso de biogás de aterros sanitários para geração de eletricidade: um estudo dos custos de implantação. In: LATIN-AMERICAN CONGRESS ELECTRICITY GENERATION AND TRANSMISSION - CLAGTEE, 11., 2015, São José dos Campos. Anais [...]. São José dos Campos: UNESP, 2015. p. 1-5.

SAYIGH, A. A. M. Comprehensive renewable energy. Amsterdã: Elsevier, 2012.

SILVA, T. N.; FREITAS, F. S. N.; CANDIANI, G. Avaliação das emissões superficiais do gás de aterros sanitários de grande porte. Engenharia Sanitária e Ambiental, Rio de Janeiro, v. 18, n. 2, p. 95-104, 2013. https://doi. org/10.1590/S1413-41522013000200001

SILVA, T. R. S.; BARROS, R. M.; TIAGO FILHO, G. L.; SANTOS, I. F. S. Methodology for the determination of optimum power of a Thermal Power Plant (TPP) by biogas from sanitary landfill. Waste Management, v. 65, p. 75-91, 2017. https://doi.org/10.1016/j.wasman.2017.04.018
SILVA, W. M. F. Consórcios públicos na gestão de resíduos sólidos urbanos no Brasil. Dissertação (Mestrado em Gestão Econômica do Meio Ambiente) - Universidade de Brasília, Brasília, 2015

SISTEMA NACIONAL DE INFORMAÇÕES SOBRE SANEAMENTO (SNIS) Série Histórica. SNIS. Disponível em: http://www.snis.gov.br/aplicacao-webserie-historica. Acesso em: 20 ago. 2019.

SUZUKI, J. A. N.; GOMES, J. Consórcios intermunicipais para a destinação de RSU em aterros regionais: Estudo prospectivo para os municípios no Estado do Paraná. Engenharia Sanitaria e Ambiental, Rio de Janeiro, v. 14 n. 2. p. 155-158, 2009. https://doi.org/10.1590/S1413-41522009000200002

VÖGELI, Y.; LOHRI, C. R.; GALLARDO, A.; DIENER, S.; ZURBRÜGG, C. Anaerobic digestion of biowaste in developing countries: practical information and case studies. Dübendorf: Swiss Federal Institute of Aquatic Science and Technology (Eawag), 2014. 137 p.

VON SPERLING, M. Princípios do tratamento biológico de águas residuárias. Belo Horizonte: Editora UFMG, 2014. 472 p.

YANG, J.; CHEN, W.; CHEN, B.; JIA, Y. Economic feasibility analysis of a renewable energy project in the rural China. Procedia Environmental Sciences, v. 13, p. 2280-2283, 2012. https://doi.org/10.1016/j.proenv.2012.01.217

ŽIŽLAVSKÝ, O. Net present value approach: method for economic assessment of innovation projects. Procedia - Social and Behavioral Sciences, v. 156, p. 506-512, 2014. https://doi.org/10.1016/j.sbspro.2014.11.230 